\title{
Plantas asociadas al turismo y los sistemas tradicionales de manejo en el occidente cercano antioqueño (Colombia)*
}

\section{Plants Associated to Tourism and Traditional Management Systems in Nearest-Western Antioquia (Colombia)}

\section{Plantes et tourisme dans le cadre des systèmes traditionnels de culture du proche Ovest du Département d'Antioquia (Colombie)}

\author{
Verónica María Álvarez O.**, Sandra B. Muriel R.***, Natalia Osorio B. ${ }^{* * * *}$
}

Recibido: 2015-03-02 // Aprobado: 2015-04-15 // Disponible en linea: 2015-06-30

Cómo citar este artículo: Álvarez, V. M., Muriel, S. B. y Osorio, N. (2015). Plantas asociadas al turismo y los sistemas tradicionales de manejo en el occidente cercano antioqueño (Colombia). Ambiente y Desarrollo, 19(37), 67-82. http://dx.doi.org/10.11144/Javeriana.ayd19-37.pats doi:10.11144/Javeriana.ayd19-37.pats

\begin{abstract}
Resumen
Las especies vegetales subvaloradas son plantas marginalmente aprovechadas, aunque presentan nutrientes esenciales valiosos. El occidente cercano antioqueño se caracteriza por el crecimiento del turismo, que ha desplazado a la producción de frutales tropicales tradicionales y con ello amenaza el conocimiento local sobre su uso y manejo. Este trabajo evaluó la diversidad de plantas con potencial de integración al turismo y describió los usos y el manejo dados por la población. Se registraron 78 especies, de las cuales el 32\% reunieron las características de ser nativas, tradicionales y aceptadas por el turismo. Las especies más promisorias fueron zapote, tamarindo, corozo, iraca y algarrobo. La alta diversidad de especies y el conocimiento tradicional deben conservarse, a través de propuestas de turismo con identidad cultural.
\end{abstract}

Palabras clave: especies marginales; conocimiento tradicional; turismo; cultivos promisorios

Este artículo está basado en los resultados del proyecto de investigación Diagnóstico sobre el manejo y uso de especies vegetales asociadas al turismo local, provenientes de huertos tradicionales del occidente cercano antioqueño, financiado por el Politécnico Colombiano Jaime Isaza Cadavid.

** Ingeniera agrónoma, Facultad de Ciencias Agrarias, Politécnico Colombiano Jaime Isaza Cadavid. Correo electrónico: veronicaalvarez@elpoli.edu.co.

Doctora en Ciencias Biológicas, Facultad de Ciencias Agrarias, Politécnico Colombiano Jaime Isaza Cadavid. Correo electrónico: sbmuriel@elpoli.edu.co.

Estudiante de Ingeniería Agropecuaria, Facultad de Ciencias Agrarias, Politécnico Colombiano Jaime Isaza Cadavid. 


\begin{abstract}
Underutilized vegetable species are plants that are marginally used, in spite of their valuable essential nutrients. Near-Western Antioquia is characterized by a growing tourism industry that has displaced the growing of traditional tropical fruits, thus threatening not only species diversity but also local wisdom on their use and handling. This work assessed plant diversity having a potential to be integrated to tourism, and described their uses and handling practices among population. 78 species were recorded, from which 32 percent met the conditions to be native, traditional and suitable for tourism. The most promising species were sapote, tamarind, "corozo2, toquilla palm, and "algarrobo", all of them currently underutilized. Plant high diversity of species and traditional knowledge on their use need to be preserved with proposals of tourism allied to cultural identity.
\end{abstract}

Keywords: marginal species; traditional knowledge; tourism; promising crops

\title{
Résumé
}

Les espèces végétales sous-estimées sont très peux exploitées et cela en dépit de leur important contenu en nutriments. Le proche Ouest du Département d'Antioquia (Colombie) connait une importante augmentation du tourisme, entrainant ainsi la diminution de la culture des fruits tropicaux traditionnels. Ce phénomène constitue une menace non seulement pour la diversité des espèces mais aussi pour le savoirfaire traditionnel des communautés de la zone. Notre travail a étudié la diversité des plantes ainsi que la possibilité de les intégrer au tourisme. II a été aussi question de décrire leurs utilisations et leur culture. Nous avons recensé 78 espèces, $32 \%$ desquelles ont été classées comme étant endémiques, traditionnelles et pouvant cohabiter avec le tourisme. Il en a résulté que les espèces avec le plus fort potentiel sont la sapotille, le tamarin, le corozo, l'iraca et le caroubier. À l'heure actuelle, toutes sont peu valorisées. Par conséquent, la grande diversité des espèces et le savoir-faire traditionnel doivent être préservés par le biais des activités touristiques avec identité culturelle.

Mots clés : espèce végétale sous-estimée; savoir-faire traditionnel; tourisme; cultures avec du potentiel 


\section{Introducción}

Las especies vegetales subvaloradas son plantas silvestres o cultivadas cuyo potencial no se ha aprovechado plenamente, incluyen variedades locales de los cultivos principales y especies actualmente abandonadas por los agricultores o en proceso de abandono (Padulosi y Hoeschle-Zeledon, 2004; Pastor et al., 2006). Muchos factores determinan la condición de marginación, olvido o subvaloración de las plantas, algunos son socioeconómicos y culturales, relacionados con el reemplazo por otras que, sesgadamente, se consideran de mayor valor (Patińo, 2002). Otros factores se relacionan con características intrínsecas de las especies, que constituyen alguna limitación para su producción comercial o uso (Pastor et al., 2006); por ejemplo, la presencia de espinas o compuestos químicos particulares (Dasgupta et al., 2013), o la corta vida en postcosecha, por lo cual su manipulación es difícil (Peña et al., 2007), entre otros.

Sobre las especies marginadas existe poca información científica y técnica disponible, no hacen parte de las agendas de investigación y desarrollo de instituciones gubernamentales, lo cual limita su proposición como cultivos novedosos (Pastor et al., 2006). Se ha demostrado que muchas de estas especies presentan componentes esenciales para una dieta adecuada, compuestos bioactivos importantes que podrían usarse en nutracéutica, farmacéutica y otras aplicaciones (Johns, 2004; Pande y Akoh, 2010).

Una consecuencia de la marginación de las especies es el riesgo de pérdida de esta diversidad y del conocimiento asociado, generado por los agricultores y sus comunidades. Este conocimiento, también conocido como "conocimiento indígena", conocimiento "local" o "cultural", representa el saber adquirido a través del contacto directo con el ambiente durante siglos, en contextos ecológicos y lingüísticos específicos (Berkes, 1993; Christensen Fund, 2014), y constituye un patrimonio intangible. El conocimiento tradicional incluye los conocimientos agrícolas, científicos, técnicos, ecológicos y medicinales y los relacionados con la diversidad biológica (Organización Mundial de la Propiedad Intelectual [OMPI], 2012), se transmite oralmente de generación en generación y su aplicación práctica se inserta en una lógica basada en la utilización de recursos alimentarios, dentro de un contexto de estrecha dependencia entre producción, disponibilidad de alimentos y consumo familiar (Velagáo, 2009). Su pérdida va contribuyendo a la pérdida de la identidad.

\section{Región de estudio}

La región del occidente cercano antioqueño incluye los municipios de San Jerónimo, Sopetrán y Santafé de Antioquia, aún se reconoce por la producción de frutales, actividad iniciada desde finales del siglo XVII y principios del siglo XVIII (Jiménez et al., 2001), bajo un modelo de agricultura tradicional diversificada (Ruiz, 2003). En muchos casos, la producción de frutales ha constituido la única fuente de ingresos para una parte de la población, a pesar de que se realiza de manera dispersa y con aplicación limitada de tecnología (Ruiz, 2003). Esta región, actualmente se denomina "el anillo turístico de occidente" (Gobernación de Antioquia, 2012a) o "la ruta del sol y las frutas" (Negret, 2010), debido a que el clima y la cercanía a Medellín favorecen las actividades de recreación y turismo.

Por efecto de la construcción de tres megaobras de importancia departamental y nacional (Gobernación de Antioquia, 2012b), se ha acelerado la presión sobre el suelo rural, por parcelaciones, actividades turísticas y fincas de recreo que han desplazado la actividad agropecuaria y forestal (Agudelo et al., 2006; Gómez y Giraldo, 2014; López, 2014). La gran diversidad de frutales que tradicionalmente ha caracterizado la región está en riesgo de pérdida y, asociado con ello, el conocimiento que los productores y la comunidad tienen sobre su manejo y uso. Por ello, este trabajo se planteó como objetivos: a) documentar el inventario de especies vegetales de importancia para el turismo y b) describir los usos y el manejo de las especies más promisorias, como un primer paso en la búsqueda de su integración a opciones de turismo planificado y de su conservación, ya que estos recursos fitogenéticos le han dado identidad a la región. 


\section{Metodología}

Esta investigación se realizó entre febrero del 2013 y septiembre del 2014, en los municipios de San Jerónimo ( $\left.6^{\circ} 26^{\prime} 29,9^{\prime \prime} \mathrm{N}, 75^{\circ} 43,3^{\prime} 38,4^{\prime \prime} \mathrm{O}\right)$, Santa Fe de Antioquia $\left(6^{\circ} 33^{\prime} 18,35^{\prime \prime} \mathrm{N}, 75^{\circ} 49,32^{\prime \prime O}\right)$ y Sopetrán $\left(6^{\circ} 30^{\prime} 21,5^{\prime \prime} \mathrm{N}, 75^{\circ} 44^{\prime 2} 24,8^{\prime \prime} \mathrm{O}\right)$ (figura 1), los cuales se ubican en la zona de vida bs-T, cuya temperatura media es $27,6^{\circ} \mathrm{C}$, su precipitación anual media $1097 \mathrm{~mm}$ y la humedad ambiental media 73,2\% (registros climáticos de 1990 a 2010, Estación Agroclimática Cotové, Ideam).

En cada municipio se establecieron contactos con organizaciones gubernamentales y no gubernamentales para identificar las familias vinculadas al turismo, a partir de la oferta de productos agrícolas en diferentes presentaciones. Se aplicó el método de muestreo intencional (Tongco, 2007), que consiste en la elección deliberada de informantes debido a su conocimiento y experiencia en un tema determinado. Se visitaron treinta fincas, en las cuales, con previa autorización de sus propietarios, se recorrieron los lotes donde se encontraban las plantas, para conocer el manejo y el estado de las plantas en los huertos.

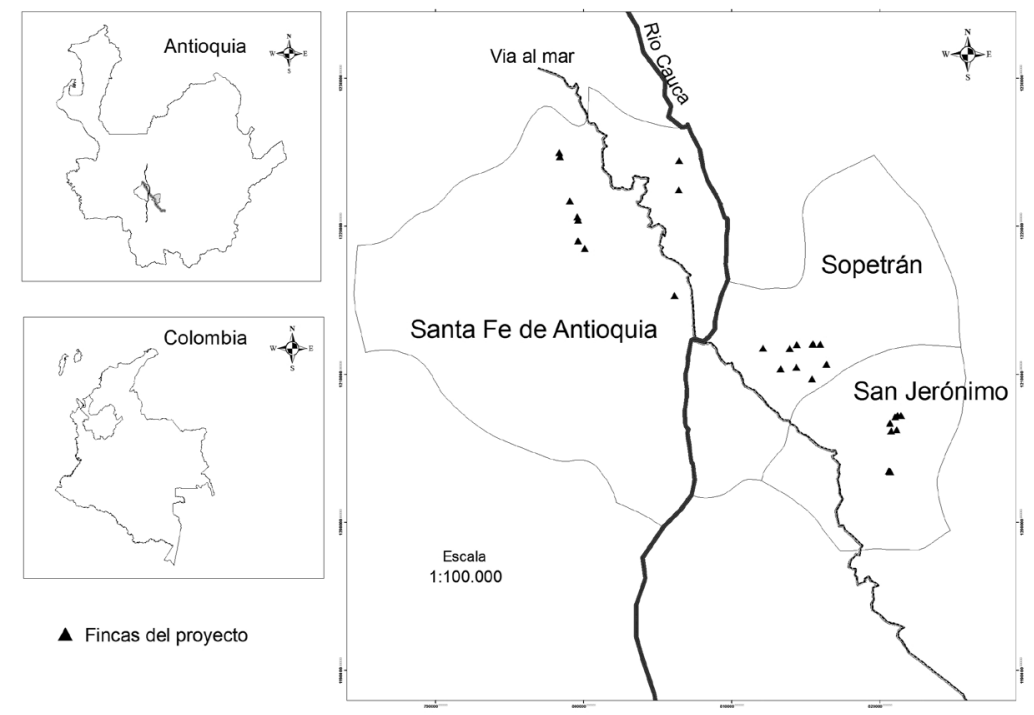

Figura 1. Localización de la región de estudio

Fuente: elaboración propia, utilizando el programa Arcgis 10.2

A través de entrevistas semiestructuradas, se indagó sobre características generales de la planta (nombre común, hábitat, hábito de crecimiento, edad, disponibilidad y calidad del material de siembra), el manejo agronómico (sistema de siembra, distribución en la parcela, distancia entre plantas, sanidad, labores culturales, cosecha, postcosecha), transformación de la parte cosechada y usos (medicinal, culinario, artesanal y otros).

A partir de la información obtenida de las fincas, se hizo un listado regional de especies. Se propuso una categorización de estas, a través de un índice de importancia (IDI) definido según la sumatoria de las seis variables cualitativas: origen de la planta (OP), grado de asociación al turismo (AT), tiempo de manejo de la planta (тм), comercialización en plazas de mercado o a través de intermediarios (м), información técnica disponible sobre la especie (IT) e iniciativas de cultivo (IC). La valoración de las variables se registra en la tabla 1. El origen de la planta varió entre nativa e introducida, para diferenciar especies originarias del trópico americano de aquellas originarias de un lugar diferente a este. 
En la variable información técnica disponible se dio un valor más alto a especies con menor información, ya que se intenta identificar las especies promisorias marginales, para darles prioridad en la investigación. La variable iniciativas de cultivo se refirió a plantas establecidas en cultivo, a diferencia de otras plantas que se siembran dispersas u otras que no se siembran, pero se aprovechan.

Tabla 1. Variables usadas para determinar el índice de importancia de plantas asociadas al turismo

\begin{tabular}{|c|c|c|}
\hline Variable & Criterio de evaluación & Valoración \\
\hline \multirow{2}{*}{ Origen de la planta (OP) } & Nativa & 1 \\
\hline & Introducida & 0 \\
\hline \multirow{2}{*}{ Asociación al turismo ( $\mathrm{AT})$} & Alta & 1 \\
\hline & Baja & 0 \\
\hline \multirow{3}{*}{ Tiempo de manejo (тм) } & Tradicional (> 60 años) & 2 \\
\hline & Intermedia (entre 20 y 59 años) & 1 \\
\hline & Nueva (< de 20 años) & 0 \\
\hline \multirow{3}{*}{ Mercado (м) } & Alto & 2 \\
\hline & Medio & 1 \\
\hline & Bajo & 0 \\
\hline \multirow{3}{*}{$\begin{array}{l}\text { Información técnica } \\
\text { disponible (Іт) }\end{array}$} & Baja & 2 \\
\hline & Media & 1 \\
\hline & Alta & 0 \\
\hline \multirow{3}{*}{ Iniciativa de cultivo (IC) } & $\begin{array}{l}\text { Plantas sembradas en parcela } \\
\text { agroforestal o cultivo limpio }\end{array}$ & 2 \\
\hline & Plantas sembradas, dispersas y cuidadas & 1 \\
\hline & $\begin{array}{l}\text { Planta no sembrada, pero cuidada y } \\
\text { aprovechada una vez se identifica }\end{array}$ & 0 \\
\hline
\end{tabular}

Fuente: elaboración propia

El resultado del iDi permitió la selección de cinco especies vegetales, acerca de las cuales se profundizó en información sobre el manejo y usos. Para analizar el manejo dado a las especies se calculó el valor de diversidad de manejo (VDM), modificado por Dos Santos et al. (2009), como el número de labores practicadas por un informante individual dividido por el número total de labores practicadas por todos los informantes. Las diferencias en el VDM entre municipios y especies se evaluaron con un test de Kruskal-Wallis (probabilidad de 5\%) y como prueba a posteriori se hizo un test de rangos múltiples. Finalmente, se hicieron entrevistas a productores y transformadores sobre los usos de las especies tradicionales, nativas y con mayor aceptación por los turistas.

\section{Resultados y discusión}

\section{Diversidad de especies en las fincas}

Se registraron 78 plantas en las treinta fincas, pertenecientes a 36 familias botánicas (tabla 2). El $44 \%$ de las familias botánicas presentaron una especie y el $25 \%$ presentaron dos especies. El $6 \%$ de las familias, correspondiente a las Anonaceae y Rutaceae, presentaron el mayor número de especies (6), les siguió la familia Fabaceae con cinco especies y luego Myrtaceae (4), Anacardiaceae (4) y Zingiberaceae 
(4) (figura 2a). Las especies con mayor IDI fueron zapote (10), tamarindo, algarrobo y corozo (9) (figura 2b). Ocuparon categorías intermedias 47 especies (5, 6 y 7 ), entre las que se encuentran frutales de gran demanda en Colombia como mango, piña, guayaba, banano y cítricos. En las categorías más bajas (3 y 4) se ubicaron cinco especies, que son introducidas, poco conocidas y/o sin mercado.

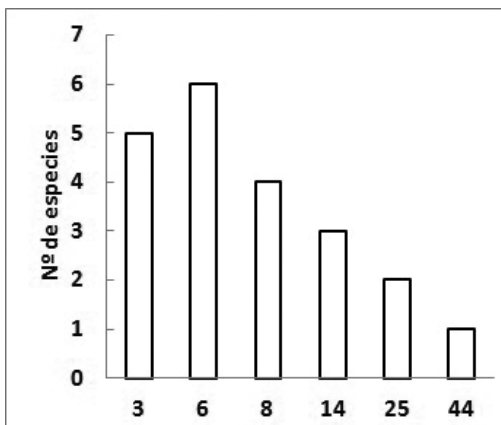

a)

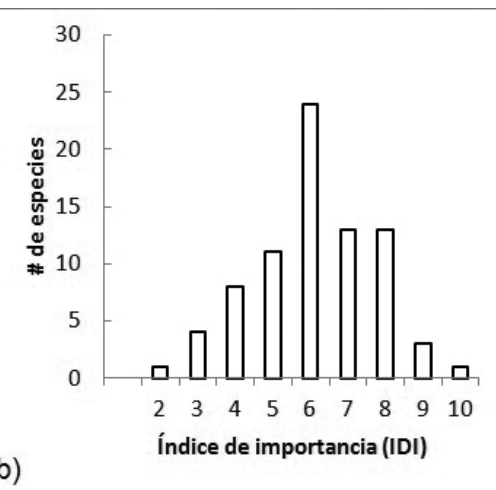

b)

Figura 2. a) Distribución de las especies inventariadas en familias botánicas y b)

Fuente: elaboración propia según el índice de importancia (IDI)

Tabla 2. Diversidad de especies vegetales del occidente cercano antioqueño, con uso actual o potencial para el turismo

\begin{tabular}{|c|c|c|c|c|c|c|}
\hline Familia & $\begin{array}{l}\text { Nombre } \\
\text { común }\end{array}$ & Nombre cientifico & $\begin{array}{c}\text { Origen * } \\
\text { (referencia) }\end{array}$ & $\begin{array}{l}\text { Asociación con } \\
\text { el turismo }\end{array}$ & $\begin{array}{l}\text { Tiempo de } \\
\text { manejo }\end{array}$ & $I D I$ \\
\hline Malvaceae & Zapote & $\begin{array}{l}\text { Matisia cordata } \\
\text { Bonpl. }\end{array}$ & $\mathrm{N}(40)$ & Alto & Tradicional & 10 \\
\hline Arecaceae & Corozo & $\begin{array}{l}\text { Aiphanes aculeata } \\
\text { Will }\end{array}$ & $\mathrm{N}(6)$ & Alto & Tradicional & 9 \\
\hline Fabaceae & Tamarindo & Tamarindus indica L. & I (20) & Alto & Tradicional & 9 \\
\hline Fabaceae & Algarrobo & $\begin{array}{l}\text { Hymenaea courbaril } \\
\text { L. }\end{array}$ & $\mathrm{N}(6)$ & Alto & Tradicional & 9 \\
\hline Annonaceae & Guanábana & Annona muricata L. & $\mathrm{N}(4)$ & Alto & Tradicional & 8 \\
\hline Caricaceae & Papaya & Carica papaya L. & $\mathrm{N}(13)$ & Alto & Tradicional & 8 \\
\hline Clusiaceae & Mamey & $\begin{array}{l}\text { Mammea americana } \\
\text { L. }\end{array}$ & $\mathrm{N}(16)$ & Alto & Tradicional & 8 \\
\hline Cucurbitaceae & Estropajo & Luffa aegyptiaca Mill. & I (18) & Alto & Tradicional & 8 \\
\hline Cyclantaceae & Iraca & $\begin{array}{l}\text { Carludovica palmata } \\
\text { Ruiz y Pav. }\end{array}$ & $\mathrm{N}(19)$ & Alto & Tradicional & 8 \\
\hline Fabaceae & Guamo & Inga spp. & $\mathrm{N}(6)$ & Alto & Tradicional & 8 \\
\hline Myrtaceae & Guayaba & Psidium guajava $\mathrm{L}$. & $\mathrm{N}(6)$ & Alto & Tradicional & 8 \\
\hline Passifloraceae & Maracuyá & $\begin{array}{l}\text { Passiflora edulis var. } \\
\text { edulis Sims. }\end{array}$ & $\mathrm{N}(27)$ & Alto & Tradicional & 8 \\
\hline Passifloraceae & Badea & $\begin{array}{l}\text { Passiflora } \\
\text { quadrangularis L. }\end{array}$ & $\mathrm{N}(27)$ & Alto & Tradicional & 8 \\
\hline Rutaceae & $\begin{array}{l}\text { Naranja } \\
\text { agria }\end{array}$ & Citrus $\times$ aurantium $\mathrm{L}$. & I (32) & Alto & Tradicional & 8 \\
\hline
\end{tabular}




\begin{tabular}{|c|c|c|c|c|c|c|}
\hline Familia & $\begin{array}{l}\text { Nombre } \\
\text { común }\end{array}$ & Nombre cientifico & $\begin{array}{c}\text { Origen * } \\
\text { (referencia) }\end{array}$ & $\begin{array}{l}\text { Asociación con } \\
\text { el turismo }\end{array}$ & $\begin{array}{l}\text { Tiempo de } \\
\text { manejo }\end{array}$ & $I D I$ \\
\hline Sapindaceae & Mamoncillo & $\begin{array}{l}\text { Melicocus bijugatus } \\
\text { Jack. }\end{array}$ & $N(6)$ & Alto & Tradicional & 8 \\
\hline Sapotaceae & $\begin{array}{l}\text { Níspero/ } \\
\text { mamey }\end{array}$ & $\begin{array}{l}\text { Manilkara zapota (L.) } \\
\text { P. Royen }\end{array}$ & N (34) & Alto & Tradicional & 8 \\
\hline Sapotaceae & $\begin{array}{l}\text { Zapote } \\
\text { costeńo }\end{array}$ & $\begin{array}{l}\text { Pouteria sapota (Jacq.) } \\
\text { H.E. Moore y Stearn }\end{array}$ & N (34) & Alto & Tradicional & 8 \\
\hline Anacardiaceae & Mango & Mangifera indica L. & I (1) & Bajo & Tradicional & 7 \\
\hline Annonaceae & $\begin{array}{l}\text { Chirimoya o } \\
\text { mamón }\end{array}$ & $\begin{array}{l}\text { Annona cherimola } \\
\text { Mill. }\end{array}$ & $\mathrm{N}(4)$ & Alto & Tradicional & 7 \\
\hline Annonaceae & Anón & Annona squamosa $\mathrm{L}$. & $\mathrm{N}(4)$ & Alto & Tradicional & 7 \\
\hline Arecaceae & Coco & Cocos nucifera L. & $\mathrm{N}(7)$ & Alto & Tradicional & 7 \\
\hline Bignoniaceae & Totumo & Crescentia cujete L. & $\mathrm{N}(8)$ & Alto & Tradicional & 7 \\
\hline Bromeliaceae & $\begin{array}{l}\text { Pińa } \\
\text { comercial }\end{array}$ & $\begin{array}{l}\text { Ananas comosus (L.) } \\
\text { Merr. }\end{array}$ & $\mathrm{N}(9)$ & Alto & Tradicional & 7 \\
\hline Chrysobalanaceae & Icaco & Chrysobalanus icaco L. & I (15) & Alto & Tradicional & 7 \\
\hline Heliconiaceae & Heliconia & $\begin{array}{l}\text { Heliconia psittacorum } \\
\text { L.f. }\end{array}$ & N (23) & Alto & Nueva & 7 \\
\hline Heliconiaceae & Heliconia & $\begin{array}{l}\text { Heliconia wagneriana } \\
\text { roja y amarilla } \\
\text { Petersen }\end{array}$ & N (23) & Alto & Nueva & 7 \\
\hline Malvaceae & Cacao & Theobroma cacao L. & $\mathrm{N}(41)$ & Bajo & Tradicional & 7 \\
\hline Rutaceae & Mandarina & $\begin{array}{l}\text { Citrus reticulata } \\
\text { Blanco }\end{array}$ & I (32) & Alto & Tradicional & 7 \\
\hline Rutaceae & Toronja & $\begin{array}{l}\text { Citrus grandis (L.) } \\
\text { Osbeck }\end{array}$ & $\mathrm{I}(32)$ & Alto & Tradicional & 7 \\
\hline Rutaceae & $\begin{array}{l}\text { Limón } \\
\text { pajarito }\end{array}$ & $\begin{array}{l}\text { Citrus limon var. } \\
\text { pajarito (L.) Osbeck }\end{array}$ & I (32) & Alto & Tradicional & 7 \\
\hline Anacardiaceae & Pistacho & Pistacia vera $\mathrm{L}$. & I (37) & Alto & Intermedia & 6 \\
\hline Anacardiaceae & Marañón & $\begin{array}{l}\text { Anacardium } \\
\text { occidentale } \mathrm{L} .\end{array}$ & $\mathrm{N}(2)$ & Alto & Intermedia & 6 \\
\hline Anacardiaceae & Ciruelo & Spondias purpurea L. & $\mathrm{N}(3)$ & Alto & Tradicional & 6 \\
\hline Arecaceae & $\begin{array}{l}\text { Palma } \\
\text { chonta }\end{array}$ & $\begin{array}{l}\text { Acrocomia aculeata } \\
\text { (Jacq.) Lodd. ex Mart }\end{array}$ & $\mathrm{N}(6)$ & Alto & Tradicional & 6 \\
\hline Bixaceae & Achiote & Bixa orellana L. & $\mathrm{N}(41)$ & Bajo & Tradicional & 6 \\
\hline Bromeliaceae & Piñuela & Bromelia karatas L. & N (9) & Alto & Tradicional & 6 \\
\hline Bromeliaceae & $\begin{array}{l}\text { Piña } \\
\text { silvestre/ } \\
\text { salvaje }\end{array}$ & $\begin{array}{l}\text { Ananas comosus (L.) } \\
\text { Merr. }\end{array}$ & $\mathrm{N}(9)$ & Bajo & Tradicional & 6 \\
\hline Cucurbitaceae & Melón & Cucumis melo L. & I (18) & Alto & Intermedia & 6 \\
\hline Cucurbitaceae & Sandía & $\begin{array}{l}\text { Citrullus lanatus } \\
\text { (Thunb.) Matsum. y } \\
\text { Nakai }\end{array}$ & I (18) & Alto & Intermedia & 6 \\
\hline Fabaceae & Cañafístula & Cassia fistula Benth. & I (21) & Alto & Tradicional & 6 \\
\hline
\end{tabular}




\begin{tabular}{|c|c|c|c|c|c|c|}
\hline Familia & $\begin{array}{l}\text { Nombre } \\
\text { común }\end{array}$ & Nombre cientifico & $\begin{array}{c}\text { Origen }{ }^{*} \\
\text { (referencia) }\end{array}$ & $\begin{array}{l}\text { Asociación con } \\
\text { el turismo }\end{array}$ & $\begin{array}{l}\text { Tiempo de } \\
\text { manejo }\end{array}$ & $I D I$ \\
\hline Marantaceae & Biao & $\begin{array}{l}\text { Calathea lutea (Aubl.) } \\
\text { Schult. }\end{array}$ & $\mathrm{N}(42)$ & Bajo & Tradicional & 6 \\
\hline Musaceae & Exótica & $\begin{array}{l}\text { Musa coccinea } \\
\text { Andrews }\end{array}$ & I (38) & Alto & Nueva & 6 \\
\hline Myrtaceae & Arazá & $\begin{array}{l}\text { Eugenia stipitata } \\
\text { McVaugh }\end{array}$ & $\mathrm{N}(6)$ & Alto & Nueva & 6 \\
\hline Oxalidaceae & Carambolo & $\begin{array}{l}\text { Averrhoa carambola } \\
\text { L. }\end{array}$ & I (26) & Alto & Tradicional & 6 \\
\hline Passifloraceae & Curucuyá & Passiflora sp. & $\mathrm{N}(27)$ & Bajo & Tradicional & 6 \\
\hline Phyllanthaceae & Grosella & $\begin{array}{l}\text { Phyllanthus acidus } \\
\text { (L.) Skeels }\end{array}$ & I (28) & Alto & Tradicional & 6 \\
\hline Poaceae & Caña brava & $\begin{array}{l}\text { Gynerium sagittatum } \\
\text { (Aubl.) P. Beauv. }\end{array}$ & N (29) & Alto & Tradicional & 6 \\
\hline Polygonaceae & Amarraboyo & Triplaris sp. & $\mathrm{N}(6)$ & Alto & Tradicional & 6 \\
\hline Rubiaceae & Noni & Morinda citrifolia L. & $\mathrm{N}(31)$ & Alto & Nueva & 6 \\
\hline Rutaceae & Pomelo & $\begin{array}{l}\text { Citrus maxima } \\
\text { (Burm.) Merr. }\end{array}$ & I (32) & Alto & Intermedia & 6 \\
\hline Vitaceae & Uva & Vitis vinifera $\mathrm{L}$. & I (36) & Alto & Intermedia & 6 \\
\hline Zingiberaceae & Exóticas & $\begin{array}{l}\text { Alpinia purpurata } \\
\text { (Vieill.) K. Schum. }\end{array}$ & I (12) & Alto & Nueva & 6 \\
\hline Zingiberaceae & Exóticas & $\begin{array}{l}\text { Etlingera elatior } \\
\text { (Jack) R.M. Sm. }\end{array}$ & I (12) & Alto & Nueva & 6 \\
\hline Zingiberaceae & Exóticas & $\begin{array}{l}\text { Zingiber sp. (var. } \\
\text { indonesia) }\end{array}$ & I (12) & Alto & Nueva & 6 \\
\hline Cactaceae & Pitaya roja & $\begin{array}{l}\text { Hylocereus undatus } \\
\text { (Haw.) Britton y } \\
\text { Rose }\end{array}$ & $\mathrm{N}(10)$ & Alto & Tradicional & 5 \\
\hline Caricaceae & $\begin{array}{l}\text { Papayuelo/ } \\
\text { tapaculo }\end{array}$ & Vasconcellea sp. & N (14) & Bajo & Tradicional & 5 \\
\hline Clusiaceae & Madrońo & $\begin{array}{l}\text { Garcinia madruno } \\
\text { (Kunth) }\end{array}$ & $\mathrm{N}(17)$ & Alto & Nueva & 5 \\
\hline Fabaceae & $\begin{array}{l}\text { Piñón de } \\
\text { oreja }\end{array}$ & $\begin{array}{l}\text { Enterolobium } \\
\text { cyclocarpum (Jacq.) } \\
\text { Griseb. }\end{array}$ & $\mathrm{N}(22)$ & Bajo & Tradicional & 5 \\
\hline Lecythidaceae & Membrillo & Gustavia augusta & $N(6)$ & Bajo & Tradicional & 5 \\
\hline Myrtaceae & $\begin{array}{l}\text { Guayaba } \\
\text { ácida o de } \\
\text { monte }\end{array}$ & Psidium araca Raddi & $\mathrm{N}(6)$ & Bajo & Tradicional & 5 \\
\hline Poaceae & Guadua & $\begin{array}{l}\text { Guadua angustifolia } \\
\text { Kunth }\end{array}$ & $N(6)$ & Bajo & Intermedia & 5 \\
\hline Punicaceae & Granada & Punica granatum L. & $\mathrm{I}(30)$ & Bajo & Tradicional & 5 \\
\hline Rubiaceae & Borojó & $\begin{array}{l}\text { Alibertia patinoi } \\
\text { (Cuatrec.) Delprete y } \\
\text { C.H. Perss. }\end{array}$ & $\mathrm{N}(31)$ & Alto & Nueva & 5 \\
\hline
\end{tabular}




\begin{tabular}{|c|c|c|c|c|c|c|}
\hline Familia & $\begin{array}{l}\text { Nombre } \\
\text { común }\end{array}$ & Nombre cientifico & $\begin{array}{c}\text { Origen * } \\
\text { (referencia) }\end{array}$ & $\begin{array}{l}\text { Asociación con } \\
\text { el turismo }\end{array}$ & $\begin{array}{l}\text { Tiempo de } \\
\text { manejo }\end{array}$ & IDI \\
\hline Rutaceae & $\begin{array}{l}\text { Azahar de la } \\
\text { India }\end{array}$ & $\begin{array}{l}\text { Murraya paniculata } \\
\text { (L.) Jack }\end{array}$ & I (33) & Bajo & Nueva & 5 \\
\hline Vitaceae & Uva silvestre & Vitis sp. & I (36) & Bajo & Tradicional & 5 \\
\hline Zingiberaceae & Cardamomo & $\begin{array}{l}\text { Elettaria } \\
\text { cardamomum (L.) } \\
\text { Maton }\end{array}$ & N (37) & Alto & Intermedia & 5 \\
\hline Annonaceae & $\begin{array}{l}\text { Noni } \\
\text { gigante }\end{array}$ & Annona glabra L. & $\mathrm{N}(5)$ & Bajo & Nueva & 4 \\
\hline Annonaceae & Anonaceae & $n . n$. & & Bajo & Nueva & 4 \\
\hline Cactaceae & Higo & Opuntia sp. & $\mathrm{I}(11)$ & Bajo & Tradicional & 4 \\
\hline Combrataceae & Almendro & Terminalia catappa L. & I (6) & Bajo & Tradicional & 4 \\
\hline Moraceae & $\begin{array}{l}\text { Árbol del } \\
\text { pan }\end{array}$ & $\begin{array}{l}\text { Artocarpus altilis } \\
\text { (Parkinson) Fosberg }\end{array}$ & I (6) & Bajo & Tradicional & 4 \\
\hline Myrtaceae & $\begin{array}{l}\text { Cerezo/ } \\
\text { pitanga }\end{array}$ & Eugenia uniflora & $\mathrm{N}(24)$ & Bajo & Nueva & 4 \\
\hline Orchidiaceae & Vainilla & Vanilla planiflora L. & I $(25)$ & Bajo & Nueva & 4 \\
\hline Sapindaceae & $\begin{array}{l}\text { Bien me } \\
\text { sabe }\end{array}$ & $\begin{array}{l}\text { Blighia sapida K.D. } \\
\text { Koenig }\end{array}$ & I (6) & Bajo & Tradicional & 4 \\
\hline Annonaceae & $\begin{array}{l}\text { Guanábana } \\
\text { africana }\end{array}$ & $n . n$ & & Bajo & Nueva & 3 \\
\hline Cannaceae & Achira & Canna indica $\mathrm{L}$. & $\mathrm{N}(12)$ & Bajo & Tradicional & 3 \\
\hline Salicaceae & $\begin{array}{l}\text { Ciruelo/ } \\
\text { Urundumbo }\end{array}$ & $\begin{array}{l}\text { Dovyalis hebecarpa } \\
\text { (Gardner) Warb. }\end{array}$ & I (39) & Bajo & Nueva & 3 \\
\hline Sapotaceae & $\begin{array}{l}\text { Fruta } \\
\text { milagrosa }\end{array}$ & $\begin{array}{l}\text { Synsepalum dulcificum } \\
\text { (Schumach. y } \\
\text { Thonn.) Daniell }\end{array}$ & I (35) & Bajo & Nueva & 3 \\
\hline
\end{tabular}

*N: nativa, l: introducida

1: Baraona y Sancho (1991); 2: Johnson (1973); 3: Acevedo y García (2012); 4: Bonavia et al. (2004); 5: Lora et al. (2011); 6: Varon y Morales (2013); 7: Gunn (2004); 8: Donkin (1974); 9: Barfuss et al. (2005); 10: Bauer (2003); 11: Grant y Grant (1979); 12: Simpson (2010); 13: Jayavalli et al. (2011); 14: Antunes y Renner (2012); 15: Barbosa et al. (2006); 16: Campbell (2005); 17: Names (2012); 18: Kocyan et al. (2007); 19: Tomlinson y Wilder (1984); 20: Tapia et al. (2012); 21: Rojas y Torres (2012); 22: Francis (1988); 23: Dobkin (1984); 24: Fao (1986); 25: Swamy (1947); 26: Núñez y Crane (2000); 27: Ocampo et al. (2007); 28: Rahman et al. (2011); 29: Suarez et al. (2009); 30: Morton (1987); 31 : McClatchey (2002); 32: Mabberley (1997); 33: Casado et al. (2011); 34: Moore y Stearn (1967); 35: Inglett y May (1968); 36: Bombardelli y Morazzoni (1995); 37: Parfitt y Badenes (1997); 38: Liu et al. (2002); 39: Lucena y Geraldo (2005); 40: Shanley et al. (2012); 41: Schultes (1979).

Fuente: elaboración propia

El 59\% de las especies inventariadas son nativas y el 32\% reúnen las características de ser nativas, tradicionales y altamente aceptadas por el turismo. Dentro de este grupo, seis especies (guanábana, guayaba, coco, piña, maracuyá y papaya) se cultivan y tienen buen mercado. Las diecinueve especies restantes son promisorias, con baja información técnica disponible; estas podrían ser seleccionadas como especies emblemáticas para ser integradas en propuestas de turismo con identidad, donde se resalte la diversidad de frutos tropicales poco comunes que pueden ser ofertados a los turistas, con la transmisión del conocimiento sobre su historia, uso y manejo. 
De las cinco especies que presentaron el mayor IDI (zapote, tamarindo, algarrobo, corozo e iraca) (tabla 2), el tamarindo es la única especie introducida, que a su vez fue priorizada, ya que se tiene hace más de doscientos años en las fincas y veredas de la región (Jiménez et al., 2001). Se evidencia su importancia local, ya que muchos lugares y negocios de alta afluencia de turistas tienen su nombre y en Santa Fe de Antioquia se realizan las Fiestas del Tamarindo. Aunque la iraca obtuvo un IDI igual al de otras doce especies (8), fue seleccionada, debido a que fue la única presente en el $100 \%$ de las fincas visitadas y a la gran diversidad de usos atribuidos por la comunidad. Sin embargo, el idi refleja que esta especie no se cultiva, y a pesar de su potencial artesanal, el mercado actual directo al turista es bajo.

\section{Uso de las especies}

El 68\% de las plantas inventariadas se ofertan a los turistas, algunas de las especies que no se ofrecen podrían ser atractivas como frutas exóticas por las características particulares que presentan. Por ejemplo, la fruta milagrosa ( $S$. dulcificum) contiene miraculina, una proteína capaz de enmascarar ciertos sabores desagradables por corto tiempo (Wilken y Satiroff, 2012), y esto produce asombro entre quienes la consumen. El uso más común de las plantas más promisorias (tabla 2) fue el consumo en fresco (85\%), alimento preparado (74\%) y servicios ambientales (65\%) (figura 3). El algarrobo es la especie a la cual se le atribuyen más usos (7), le siguen el tamarindo (6) y posteriormente la iraca, el mamey y el coco (5) (figura 3).

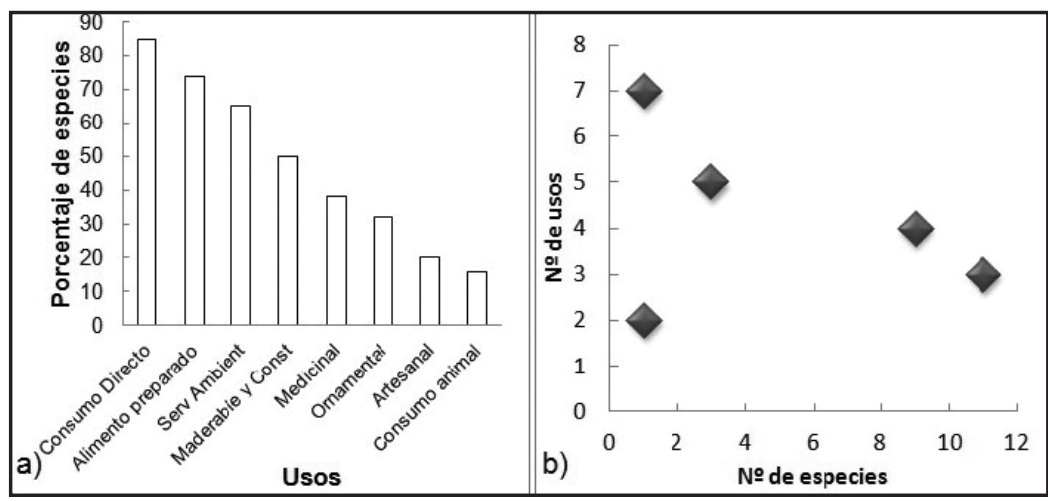

Figura 3. Usos dados por la comunidad en el occidente cercano antioqueño a las especies de mayor importancia para el turismo. a) Usos, b) número de usos

Fuente: elaboración propia

\section{Manejo de las especies priorizadas}

Las fincas evaluadas son explotadas por sus propios dueños, el $56 \%$ presentan menos de 5 ha, el $38 \%$ entre 5 y 50 ha y el $5 \%$ presentan más de 50 ha. Los propietarios nunca han recibido asistencia técnica para el manejo de las especies, sino que intercambian información del manejo con vecinos y amigos.

En los tres municipios desarrollan una agricultura tradicional de baja tecnificación, aunque hay diferencias en cuanto al VDM $(F=14,9 ; p=0,00)$ (figura 4a). Así, en Sopetrán realizan un mayor número de labores para el mantenimiento de las plantas priorizadas, como riego semanal, siembra en un vivero casero, desyerba, manejo sanitario y cosecha. En este municipio el empleo de mano de obra en un $73 \%$ es contratada, mientras en Santa Fe de Antioquia un 25\% y en San Jerónimo toda la mano de obra es familiar. En Sopetrán se hace selección y pesaje del producto cosechado en un $45 \%$ de las fincas, en San Jerónimo se selecciona en un 20\% y en Santa Fe en un 12,5\%. El manejo del agua es una de las labores que más importancia recibe, debido a las características del clima regional. 


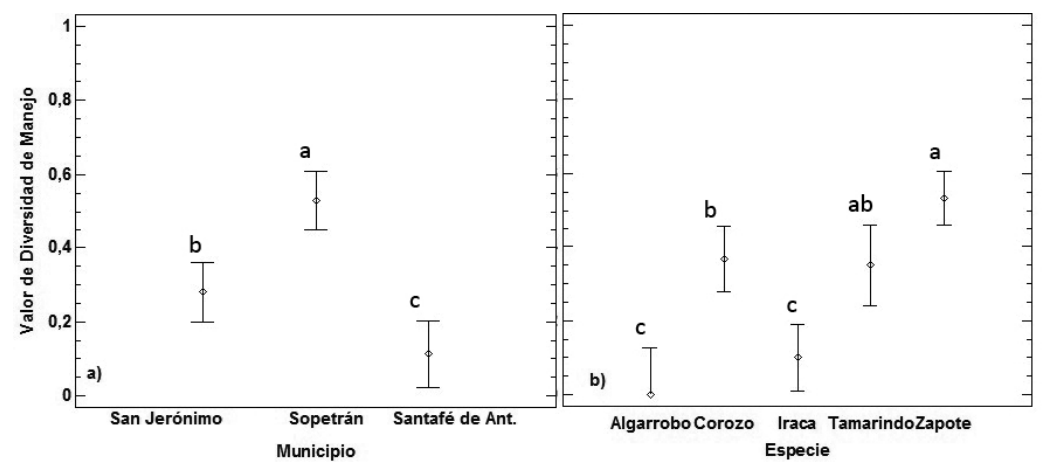

Figura 4. Comparación del valor de diversidad de manejo (VDM) a) entre municipios y b) entre especies priorizadas (letras diferentes indican diferencia mínima significativa)

\section{Fuente: elaboración propia}

Se encontró que el manejo también difiere por especie (figura $4 \mathrm{~b})(\mathrm{F}=11,25 ; p=0,00)$. El zapote es la especie con mayor número de labores, debido a su importancia para los productores, ya que tiene buen mercado. Esta especie se siembra en un sistema agroforestal multi-estrato, donde hay suministro de riego semanal. Las plantas nuevas se siembran en vivero rudimentario hecho en la propia finca, aplican fertilizantes en algunos casos y hacen selección del producto para la venta. La gente reconoce dos variedades: el zapote criollo, que manejan desde hace más de doscientos años (Jiménez et al., 2001), su fruto es pequeño y dulce. La variedad ecuatoriana es de mayor tamaño y fue introducido por los comercializadores de frutas, que lo llevaron desde las plazas de mercado de Medellín hace veinticinco años, aproximadamente.

La cosecha de los frutos se hace entre julio y septiembre y es comprada por intermediarios, quienes llevan la mayor parte directamente a las plazas de mercado de Medellín, y solo una pequeña parte de la producción se oferta a los turistas localmente. Las plantas de zapote se encontraron en los siguientes arreglos de cultivo: cacao, como cultivo principal con sombrío de zapote y otros frutales arbóreos; zapote, como cultivo principal, con plantas ornamentales de las familias Zingiberaceae y Heliconiaceae en un estrato inferior, o en un huerto tradicional con muchos frutales arbóreos mezclados, sin arreglo ni distancias de siembra.

El tamarindo es una planta de gran importancia, que tiene buena comercialización, debido a que con el fruto se elaboran dulces y néctares, los cuales se han constituido en los productos de mayor recordación por los turistas. Estos dulces son consumidos y adquiridos como souvenir, aunque su calidad debe mejorarse. En algunas épocas del año la fruta es costosa para los fabricantes de dulces, debido a su cosecha estacional (entre diciembre y febrero). En algunas fincas se tiene en arreglo silvopastoril y se hace un manejo similar al del zapote (figura $4 \mathrm{~b}$ ), aunque también se observa como árboles dispersos en caminos, potreros, huertos o patios traseros en casas urbanas que se cosechan y reciben poco manejo.

El corozo se tiene como planta dispersa (figura 4b), generalmente no se siembra, solo se cosecha, más que un sistema productivo es un sistema en el cual los agricultores actúan como recolectores en sus predios. Solo una finca ha innovado sembrando en hileras, a manera de cultivo. Los frutos se ofertan en fresco o secos; en este caso el secado se hace artesanalmente en las fincas. La iraca y el algarrobo son especies de las cuales se hace un uso extractivo, la labor más importante es la cosecha (figura 4b). La iraca generalmente está dentro de las fincas, el propietario cosecha todas las hojas cuando tiene un pedido para hacer techos principalmente, dejando solo los tallos cortos para que la planta se regenere. El algarrobo puede estar dentro de las fincas o caminos y áreas silvestres. El mercado de la harina de algarrobo ha aumentado en los últimos años entre turistas y residentes, debido a la popularidad de su uso para tratar la gastritis, el colon irritado y a su valor alimenticio (tabla 3). En algunas épocas del año su precio es atractivo para los propietarios y recolectores. 


\section{Las plantas y el turismo}

En la población existe la percepción de que el cultivo, manejo y procesamiento de estas especies va a desaparecer por la presión del uso de la tierra, y los municipios no reconocen la importancia de proteger estos recursos genéticos, ni están prepados para enfrentar los cambios y proteger el patrimonio cultural y genético que tienen.

En este estudio se encontró una alta diversidad de plantas que son atractivas para los turistas, conservadas en fincas tradicionales. Se propone la apropiación de este recurso como estrategia para fortalecer propuestas de un turismo sostenible, que se armonicen con la identidad cultural. Para ello, algunas plantas pueden ser seleccionadas como emblemáticas, si se resaltan sus cualidades de ser nativas, tradicionales y altamente vinculadas al turismo. Estas plantas, ofertadas de diversas formas: frutos frescos, alimentos procesados y artesanías, podrían ser claves en la transmisión del conocimiento local, donde se resalten los diferentes oficios, manejos, usos, recetas y formas de procesamiento. Incluso, las fincas tradicionales donde se conservan las plantas pueden ser modelos de propuestas de turismo alternativo, que se han ensayado en otros países y regiones como el agroturismo y el turismo cultural, que podrían inspirar propuestas locales (Ramírez, 2007; López, 2014).

\section{Conclusiones}

En las fincas tradicionales del bs- $T$ del occidente cercano antioqueño se identificaron 78 especies, dentro de las cuales un alto porcentaje son marginadas, nativas, compatibles con el turismo, que ameritan ser conservadas. Cinco especies son de mayor importancia: zapote, tamarindo, corozo, algarrobo e iraca. El zapote es la especie con mayor VDM y vinculación al mercado, aunque se ofrece solamente como fruto fresco; el tamarindo se identificó como una especie introducida, pero de mayor reconocimiento por los turistas y de alta diversidad de usos. El algarrobo y la iraca tienen alta diversidad de usos por las comunidades, no solamente con relación al turismo. El manejo agronómico de las especies priorizadas es escaso, aunque en Sopetrán se realizan más labores, probablemente debido a la tradición frutícola del municipio.

Las especies se ofertan a los turistas con poco valor agregado y en algunos casos los productos son de baja calidad, por lo cual es importante acompañar a las comunidades en un proceso de mejoramiento de estas actividades. Asimismo, se está perdiendo la memoria colectiva del procesamiento y uso; es el caso de la iraca como insumo artesanal tradicional.

El turismo y la amplia demanda de la tierra para establecimiento de parcelaciones son una amenaza a la cultura local, pero también podrían ser la oportunidad de revalorar el capital tangible e intangible de estos municipios y vincular estos recursos fitogenéticos a propuestas de un turismo que valore la identidad cultural, donde se conserven las especies marginadas y el conocimiento tradicional sobre ellas.

Agradecimientos: Al Politécnico Colombiano Jaime Isaza Cadavid por la financiación de este proyecto, a las instituciones gubernamentales y no gubernamentales de los municipios de San Jerónimo, Sopetrán y Santa Fe de Antioquia, propietarios de las fincas visitadas, transformadores y comercializadores. A Teresa Robledo, Sebastián Montoya, Clara I. Correa, Mariluz Aguilar y Jorge H. Madrid, y especialmente a León Darío Vélez, por sus sugerencias. 


\section{Referencias}

Acevedo, I. y García, O. (2012). Elaboración y evaluación de ciruela (Spondias purpurea L.) en almíbar como rellenos en queso tipo Mozzarella de búfala (Bubalus bubalis). Revista Cientifica UDO Agricola, 12(3), 720-729.

Antunes, F. y Renner, S. S. (2012). A Dated Phylogeny of the Papaya Family (Caricaceae) Reveals the Crop's Closest Relatives and the Family's Biogeographic History. Molecular Phylogenetics and Evolution, 65(1), 46-53.

Baraona, M. y Sancho, E. (1991). Fruticultura especial: fasículo 2. aguacate y mango. fruticultura II. San José de Costa Rica: Universidad Estatal a Distancia.

Barbosa, W. L. R., Peres, A., Gallori, S. y Vincieri, F. F. (2006). Determinação de derivados de miricetina em Chrysobalanus icaco L. Chrysobalanaceae). Revista Brasileira de Farmacognosia, 16(3), 333-337.

Barfuss, M. H., Samuel, R., Till, W. y Stuessy, T. F. (2005). Phylogenetic Relationships in Subfamily Tillandsioideae (Bromeliaceae) Based on Dna Sequence Data from Seven Plastid Regions. American Journal of Botany, 92(2), 337-351.

Bauer, R. A. (2003). Sinopsis of the tribu Hyloceraceae F. Buxb. Cactaceae Systematics Initiatives, (17), 3-4.

Berkes, F. (1993). Traditional Ecological Knowledge in Perspectives. En J. Inglis (Ed.), Traditional Ecological Knowledge: Concepts and Cases. Canada: IDRC.

Bombardelli, E. y Morazzoni, P. (1995). Vitis vinifera L. Fitoterapia, 66(4), 291-317.

Bonavia, D., Ochoa, C. M., Tovar, S. Ó. y Palomino, R. C. (2004). Archaeological Evidence of Cherimoya (Annona cherimolia Mill.) and Guanabana (Annona muricata L.) in ancient Peru. Economic Botany, 58(4), 509-522.

Campbell, R. J. (2005). Collecting Mammea americana L. in tropical America: Potential for Florida. Proceedings of the Florida State Horticultural Society, (118), 242-243.

Casado, C. M., Gutiérrez, Y. I. y Rodríguez, E. (2011). Acercamiento al género Murraya (Rutaceae) y a la especie Murraya paniculata (L.) Jack. Revista Cubana de Plantas Medicinales, 16(4), 408-418.

Christensen Fund (2014). Traditional Knowledge Transfer. Recuperado el 12 de junio del 2014, de: http://www. christensenfund.org/issues/traditional-knowledge-transfer/.

Dasgupta, P., Chakraborty, P. y Bala, N. N. (2013). Averrhoa carambola: An Updated Review. International Journal of Pharma Research \& Review, 2, 54-63.

Dobkin, D. S. (1984). Flowering Patterns of Long-Lived Heliconia Inflorescences: Implications for Visiting and Resident Nectarivores. Oecologia, 64(2), 245-254.

Donkin, R. A. (1974). Bixa Orellana: "The Eternal Shrub”. Anthropos, 33-56.

Dos Santos, K. L., Peroni, N., Guries, R. P. y Nodari, R. O. (2009). Traditional Knowledge and Management of Feijoa (Acca sellowiana) in southern Brazil. Economic Botany, 63(2), 204-214.

Food and Agriculture Organization of The United Nations (1986). 44/3 Food and fruit-bearing species Latin America. Roma.

Francis, K. (1988). Enterolobium cyclocarpum (Jacq.) Griseb. Guanacaste, Earpod-Tree. SO-ITF-SM-15. Nueva Orleans: Department of Agriculture, Forest Service, Southern Forest Experiment Station.

Gobernación de Antioquia (2012a). Lineamientos de ordenación territorial para Antioquia. Construyendo acuerdos territoriales. Recuperado el 20 enero del 2015, de:http://ordenacionterritorialantioquia.info/docs/ cartografia/estructura_ordenacion_territorial_Occidente.pdf. 
Gobernación de Antioquia (2012b). El Urabá antioqueño: un mar de oportunidades y potencialidades. Recuperado el 20 enero del 2015, de: http://www.antioquia.gov.co/Plan_de_desarrollo_2012_2015/PDD_FINAL/ PDD_FINAL/10_Linea_6.pdf.

Gómez-Vargas, A. B. y Giraldo-Calderón, P. E. (2014). Análisis relacional del sistema alimentario en los municipios de Andes y Santa Fe de Antioquia: relación entre actores, agentes e instituciones. Cuadernos de Desarrollo Rural, 11(73), 191-210.

Grant, V. y Grant, K. A. (1979). Systematics of the Opuntia phaeacantha group in Texas. Botanical Gazette, 199-207. Inglett, G. E. y May, J. F. (1968). Tropical Plants with Unusual Taste Properties. Economic Botany, 22(4), 326-331.

Gunn, B. F. (2004). The Phylogeny of the Cocoeae (Arecaceae) with Emphasis on Cocos Nucifera. Annals of the Missouri Botanical Garden, 505-522.

Jayavalli, R., Balamohan, T. N., Manivannan, N. y Govindaraj, M. (2011). Breaking the Intergeneric Hybridization Barrier in Carica papaya and Vasconcellea cauliflora. Scientia Horticulturae, 130(4), 787-794.

Jiménez O., Gutiérrez J. F., Londońo J. G. y Salazar, C. A. (2001). Configuración regional del Occidente medio de Antioquia. Siglos XVI-XVIII. Medellín: Corporación Autonoma Regional del Centro de Antioquia Universidad Nacional de Colombia.

Johns, T. (2004). Especies subutilizadas y nuevos retos para la salud global. LEISA Revista de Agroecología, 20(1), 9-10.

Johnson, D. (1973). The Botany, Origin, and Spread of the Cashew Anacardium occidentale L. Journal of Plantation Crops, 1(1-2), 1-7.

Liu, A. Z., Li, D. Z. y Li, X. W. (2002). Taxonomic Notes on Wild Bananas (Musa) from China. Botanical Bulletin of Academia Sinica, (43), 77-81.

Lora, J., Herrero, M. y Hormaza, J. I. (2011). Stigmatic Receptivity in a Dichogamous Early-Divergent Angiosperm Species, Annona cherimola (Annonaceae): Influence of Temperature and Humidity. American Journal of Botany, 98(2), 265-274.

Lucena, I. y Geraldo, A. (2005). Physical and Chemical Characterization of Dovyalis Fruits. International Journal of Fruit Science, 5(4), 39-46.

Kocyan, A., Zhang, L. B., Schaefer, H. y Renner, S. S. (2007). A Multi-Locus Chloroplast Phylogeny for the Cucurbitaceae and its Implications for Character Evolution and Classification. Molecular Phylogenetics and Evolution, 44(2), 553-577.

Mabberley, D. J. (1997). A Classification for Edible Citrus (Rutaceae).Telopea, 7(2), 167-172.

McClatchey, W. (2002). From Polynesian Healers to Health Food Stores: Changing Perspectives of Morinda citrifolia (Rubiaceae). Integrative Cancer Therapies, 1(2), 110-120.

Moore Jr, H. E. y Stearn, W. T. (1967). The Identity of Achras zapota L. and the Names for the Sapodilla and the Sapote. Taxon, 16(2), 382-395. doi: 10.2307/1216409

Morton, J. F. (1987). Fruits of Warm Climates. Recuperado el 20 de enero del 2015, de https://www.hort.purdue. edu/newcrop/morton/pomegranate.html.

Motamayor, J. C., Risterucci, A. M., López, P. X. A., Ortiz, C. X. F., Moreno, A. y Lanaud, C. (2002). Cacao Domestication I: the Origin of the Cacao Cultivated by the Mayas. Heredity, 89(5), 380-386.

Names, V. (2012). Garcinia madruno. Edible Medicinal And Non-Medicinal Plants, 2(2), 76.

Negret, J. 2010. Libro de estadísticas de turismo 2010. Sistema de Indicadores Turisticos Medellín: SITUR. Recuperado el 20 de enero del 2015, de http://situr.gov.co/images/boletines/libroestadistica2010.pdf 
Núñez, R. y Crane, J. H. (2000). Selective Pruning and Crop Removal Increase Early-Season Fruit Production of Carambola (Averrhoa carambola L.). Scientia Horticulturae, 86(2), 115-126.

Ocampo J., d'Eeckenbrugge, G. C., Restrepo, M., Jarvis, A., Salazar, M. y Caetano, C. (2007). Diversity of Colombian Passifloraceae: Biogeography and an Updated List for Conservation. Biota Colombiana, $8(1), 1-45$.

Organización Mundial de la Propiedad Intelectual (OMPI) (2012). Guía de la organización mundial de la propiedad intelectual para la catalogación de conocimientos tradicionales. Ginebra: wIPO.

Padulosi, S. y Hoeschle-Zeledon, I. (2004). Underutilized Plant Species: What Are They? LEISA Magazin, (20), 5-6.

Pande, G. y Akoh, C. (2010). Organic Acids, Antioxidant Capacity, Phenolic Content and Lipid Characterisation of Georgia-Grown Underutilized Fruit Crops. Food Chemistry, 120, 1067-1075.

Parfitt, D. E. y Badenes, M. L. (1997). Phylogeny of the genus Pistacia as Determined from Analysis of the Chloroplast Genome. Proceedings of the National Academy of Sciences, 94(15), 7987-7992.

Pastor, S., Fuentealba, B. y Ruiz, M. (2006). Underutilised Crops in Perú: Some Conceptual and Political Considerations. Recuperado el 20 de enero del 2015, de http://www.underutilized-species.org/Documents/PubliCATIONs/ underutilized_species_policies_and_strategies_peru_lr.pdf.

Patińo, V. M. (2002). Historia y dispersión de los frutales nativos del neotrópico (nº 326). Cali: Ciat, Asohofrucol, Fondo Nacional de Fomento Hortifrutícola.

Peña, A. C., González, M. L., Hernández, M. S., Novoa, C., Quicazán, M. C. y Fernández-Trujillo, J. P. (2007). Evaluación del comportamiento del fruto de arazá (Eugenia stipitata Mc Vaugh) en las operaciones de acondicionamiento húmedo poscosecha. Revista Colombiana de Ciencias Hortícolas, 1(2), 182-188.

Rahman, M. M., Habib, M. R., Hasan, S. R., Aktar, M. y Sayeed, R. M. (2011). Antibacterial, Cytotoxic and Antioxidant Potential of Methanolic Extract of Phyllanthus acidus L. International Journal of Drug Development and Research, 3(2), 154-161.

Ramírez, C.R. (2007). Etnobotánica y la pérdida de conocimiento tradicional en el siglo 21. Ethnobotany Research \& Applications, (5), 241-244.

Rojas, F. y Torres, G. (2012). Árboles del Valle Central de Costa Rica: reproducción Caña Fístula (Cassia fistula L.). Revista Forestal Mesoamericana Kurú, 9(23), 49-50.

Ruiz M. N. (2003). El occidente antioqueño: perfil subregional. Dirección planeación estratégica integral. Medellín: Departamento Administrativo de Planeación de Antioquia.

Schultes, R. E. (1979). The Amazonia as a Source of New Economic Plants. Economic Botany, 33(3), 259-266.

Simpson, M. G. (2010). Plant systematics. Estados Unidos: Academic Press.

Shanley, P., Cymerys, M., Serra, M. y Medina, G. (2012). Frutales y plantas utiles en la vida amazonica. Productos forestales no madereros. Roma: FAO.

Suárez, I. E., Araméndiz, H. y Pastrana, I. J. (2009). Micropropagation of Wild Cane (Gynerium sagitatum Aubl.). Revista-Facultad Nacional de Agronomia, 62(2), 5135-5143.

Swamy, B. G. L. (1947). On the Life-History of Vanilla Planifolia. Botanical Gazette, 449-456.

Tapia, F., Mercado, P. y Gómez, S. (2012). Contribución a la citogenética de Tamarindus indica (Leguminosae: Caesalpinioideae). Acta Botánica Mexicana, (98), 99-110.

Tomlinson, P. B. y Wilder, G. J. (1984). Systematic Anatomy of Cyclanthaceae (Monocotyledoneae)-An Overview. Botanical Gazette, 535-549. 
Tongco, M. D. C. (2007). Purposive Sampling as a Tool for Informant Selection. Ethnobotany Research y Applications, (5), $147-158$

Valagão M. M. (2009). La sopa y las sopas de plantas silvestres alimentarias en El Alentejo, Portugal. Food, Imaginaries and Cultural Frontiers Essays in Honour of Helen Macbeth. México: Universidad de Guadalajara, Colección Estudios del Hombre, Serie Antropología de la Alimentación (vol. 24).

Varon, T. y Morales, L. (2013). Arboretum y Palmetum: Guía de identificación. Medellín: Universidad Nacional de Colombia.

Wilken, M. K. y Satiroff, B. A. (2012). Pilot Study of "Miracle Fruit" to Improve Food Palatability for Patients Receiving Chemotherapy. Clinical Journal of Oncology Nursing, 16(5), E173-E177. 